A. K. Dupree and A. O. Benz, Eds.

\title{
The Photospheric Abundances of Active Binaries: First Results
}

\author{
T. Morel, G. Micela \\ INAF, Osservatorio Astronomico di Palermo G.S. Vaiana, Italy \\ F. Favata \\ Astrophysics Division ESA, ESTEC, Noordwijk, The Netherlands \\ D. Katz
}

Observatoire de Paris, GEPI, Place Jules Janssen, Meudon, France

\section{Pillitteri}

\section{Università di Palermo, Italy}

\begin{abstract}
Photospheric parameters and abundances of 13 chemical species are presented for a sample of single-lined chromospherically active binaries from a differential LTE analysis of high-resolution spectra. Our results indicate that the X-ray active binaries studied are not as metal poor as previously claimed, but are at most mildly iron-depleted relative to the Sun $(-0.41 \lesssim[\mathrm{Fe} / \mathrm{H}] \lesssim+0.11)$. A significant overabundance of several elements (e.g., $\mathrm{Na}, \mathrm{Mg}, \mathrm{Al}, \mathrm{Ca}$ ) is observed. The temperatures derived from the $(V-R)$ and $(V-I)$ colours are found to be significantly affected by activity processes.
\end{abstract}

\section{Introduction}

We have recently embarked on a project that aims at deriving photospheric parameters and metal abundances for a large sample of active binaries (see Katz et al. 2003 for the first results). This will help, for instance, to better characterize the evolutionary status of this population and the chemical fractionation processes operating between the photosphere and the corona. We refer the reader to Morel et al. (2003) for more details regarding the results presented here.

\section{Observations}

Spectra of 28 active binary systems (drawn from the list of Strassmeier et al. 1993) were acquired in January 2000 at the ESO $1.52-\mathrm{m}$ telescope at La Silla (Chile) with the echelle spectrograph FEROS. The spectral range covered is $3600-9200 \AA$, with a resolving power of 48000 . Here we present a curve-ofgrowth abundance analysis of 6 single-lined systems (all of spectral type K0-K2 
IV) with a modest projected rotational velocity $\left(v \sin i \lesssim 10 \mathrm{~km} \mathrm{~s}^{-1}\right)$. The data for the more rapid rotators will be analized by means of other techniques (e.g., spectral synthesis) because of severe blending problems.

\section{Methodology and results}

The photospheric parameters $\left(T_{\text {eff }}, \log g\right.$, and $\left.\xi\right)$ and metal abundances were derived by using the measured equivalent widths and a set of Kurucz atmospheric models as input for the MOOG software. Two methods have been used. The abundances were first derived from a fully self-consistent analysis of the spectra, whereby the temperature is determined from the excitation equilibrium of the Fe I lines (hereafter Method 1). The second approach (hereafter Method 2) relies on temperatures derived from the empirical $T_{\text {eff }}-(B-V)$ calibration of Alonso, Arribas, \& Martínez-Roger (1999). In both cases, the surface gravity is derived from the ionization equilibrium of the Fe lines and the microturbulent velocity by requiring the iron abundance to be independent of the line strength.

The two methods described above yield in some cases significantly different values for the effective temperatures and surface gravities, but the abundance patterns are generally very similar (Fig.1). The stars studied exhibit significant overabundances of most elements with respect to the solar pattern (up to 0.5 dex). There is a clear indication for an increasing abundance of the $\alpha$-elements with decreasing $[\mathrm{Fe} / \mathrm{H}]$ (see Fig.2). We compare in Figure 3, as a function of several activity indicators, the effective temperatures derived from the excitation equilibrium of the Fe I lines and from the $(B-V),(V-R)$, and $(V-I)$ indices. There is a suggestive indication, in particular from the Ca II $\mathrm{H}+\mathrm{K}$ data, that the temperature discrepancy increases with the stellar activity level.

\section{Conclusions}

The $(B-V)$ index appears to be a more robust indicator of the effective temperature in chromospherically active binaries than $(V-R)$ and $(V-I)$. An a priori knowledge of the metallicity is, however, required. Our results suggest that the active binaries studied may not be as iron-deficient as previously thought (Randich, Gratton, \& Pallavicini 1993; Randich, Giampapa, \& Pallavicini 1994). The physical mechanisms leading to the noticeable overabundance of most elements with respect to iron remain to be identified (Figs.1-2), although an interpretation in terms of nucleosynthesis yields remains viable. In particular, the gradient between $[\alpha / \mathrm{Fe}]$ and $[\mathrm{Fe} / \mathrm{H}]$ is much steeper than for thin disk dwarfs (e.g., Reddy et al. 2003), but is reminiscent of the thick disk population (e.g., Feltzing, Bensby, \& Lundström 2003). Our data do not show a clear correlation between the abundance ratios and the activity level. Firm conclusions regarding the potential role played by chromospheric activity in inducing the overabundances observed must therefore await the analysis of a much larger sample, along with a detailed theoretical investigation. 


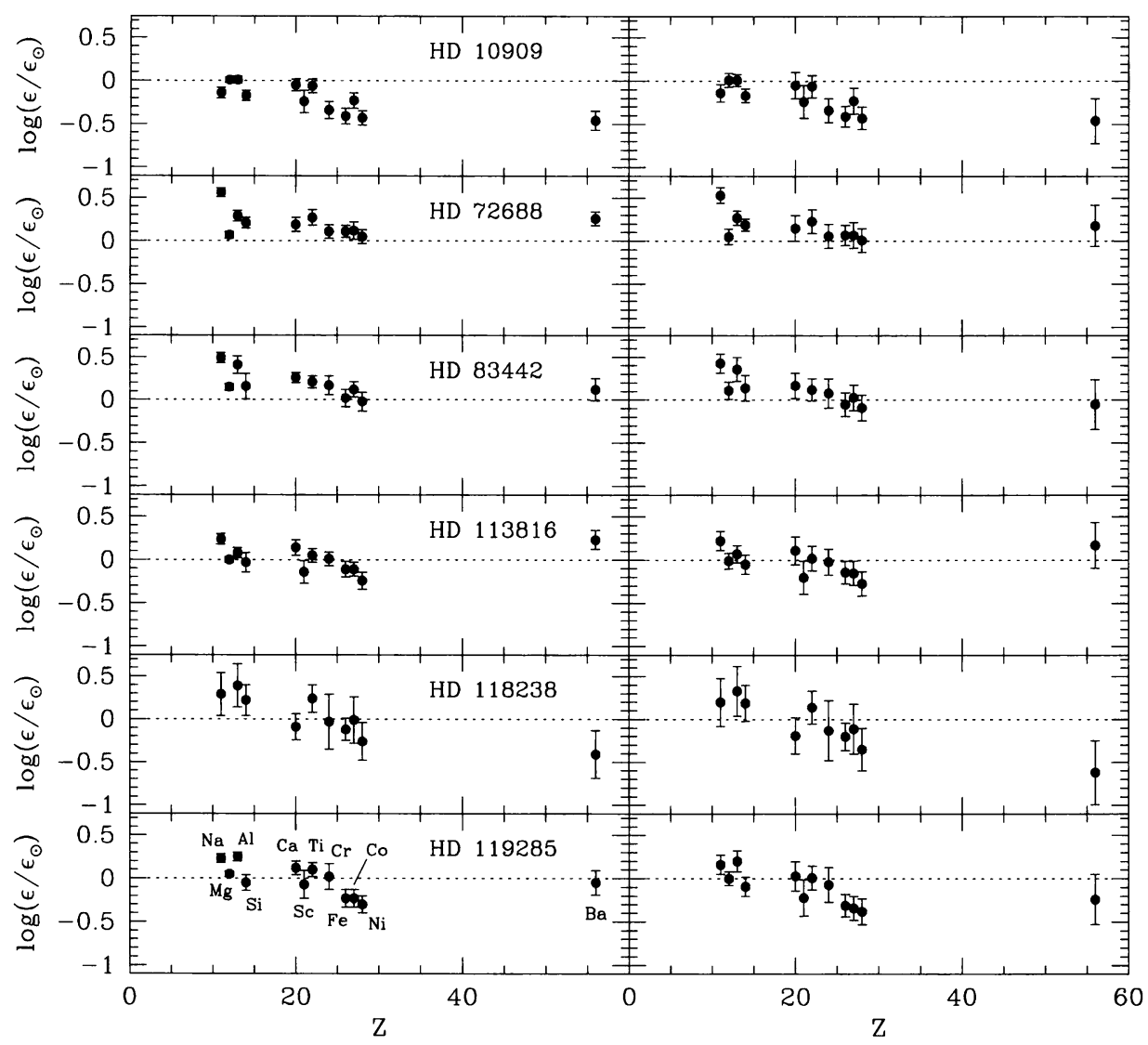

Figure 1. Abundance patterns for the stars in our sample determined from Method 1 (left-hand panels) and Method 2 (right-hand panels). The abundances of $\mathrm{Na}, \mathrm{Mg}$ and $\mathrm{Ca}$ have been corrected for departures from LTE (Drake 1991; Gratton et al. 1999).

\section{References}

Alonso, A., Arribas, S., \& Martínez-Roger, C. 1999, A\&AS, 140, 261

Dempsey, R. C., Linsky, J. L., Fleming, T. A., \& Schmitt, J. H. M. M. 1993, ApJS, 86, 599

Dempsey, R. C., Linsky, J. L., Fleming, T. A., \& Schmitt, J. H. M. M. 1997, ApJ, 478, 358

Drake, J. J. 1991, MNRAS, 251, 369

Feltzing, S., Bensby, T., \& Lundström, I. 2003, A\&A, 397, L1

Gratton, R. G., Carretta, E., Eriksson, K., \& Gustafsson, B. 1999, A\&A, 350, 955

Katz, D., Favata, F., Aigrain, S., \& Micela, G. 2003, A\&A, 397, 747 

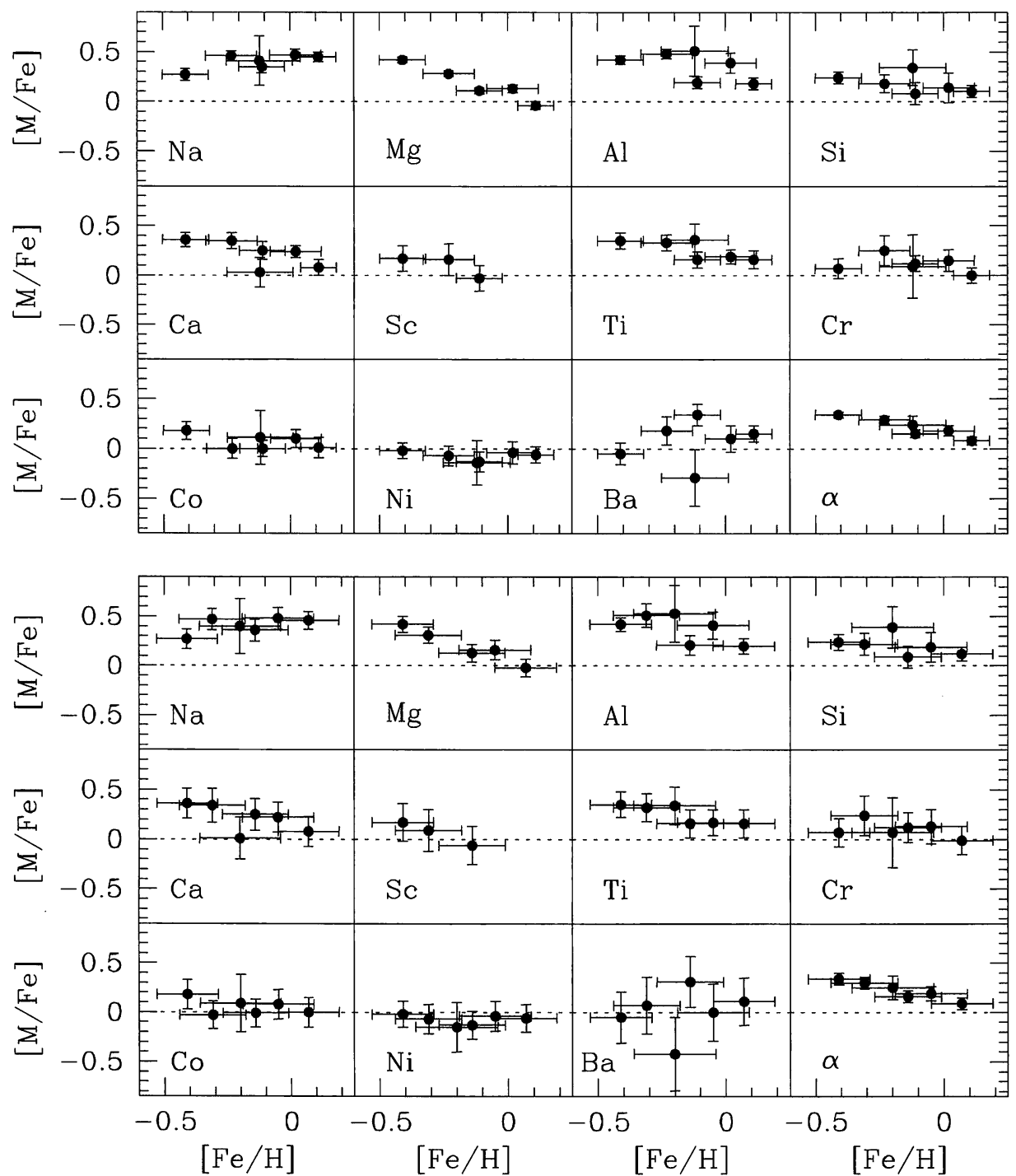

Figure 2. Abundance ratios as a function of $[\mathrm{Fe} / \mathrm{H}]$ for Method 1 (top) and Method 2 (bottom). The abundances of $\mathrm{Na}, \mathrm{Mg}$ and $\mathrm{Ca}$ have been corrected for departures from LTE (Drake 1991; Gratton et al. 1999). We define $[\alpha / \mathrm{Fe}]$ as the mean of the $\mathrm{Mg}, \mathrm{Si}, \mathrm{Ca}$, and $\mathrm{Ti}$ abundance ratios.

Morel, T., Micela, G., Favata, F., Katz, D., \& Pillitteri, I. 2003, A\&A, 412, 495 Randich, S., Gratton, R., \& Pallavicini, R. 1993, A\&A, 273, 194

Randich, S., Giampapa, M. S., \& Pallavicini, R. 1994, A\&A, 283, 893 

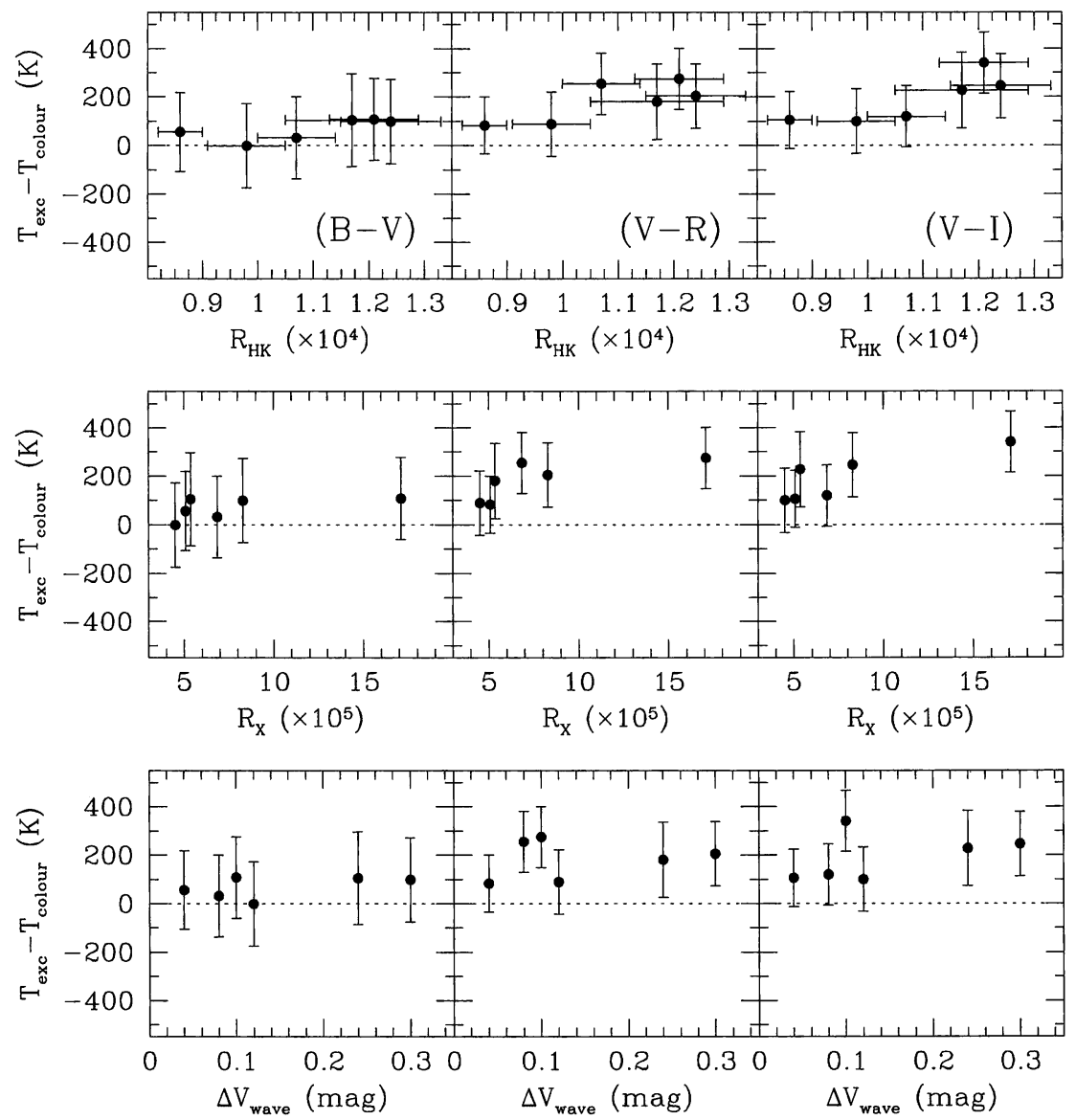

Figure 3. Differences between the excitation and colour temperatures, as a function of the activity indices $R_{\mathrm{HK}}$ (top panels), $R_{\mathrm{X}}$ (middle panels), and $\Delta V_{\mathrm{w}}$ ve (bottom panels). $R_{\mathrm{HK}}$ and $R_{\mathrm{X}}$ are defined as the radiative loss in the Ca II $\mathrm{H}+\mathrm{K}$ lines and the $\mathrm{X}$-ray luminosity (0.1-2.4 $\mathrm{keV}$ ) both in units of the bolometric luminosity, respectively (X-ray data from Dempsey et al. 1993, 1997). $\Delta V_{\mathrm{w}}$ ve is an indicator of stellar spottedness, and is defined as the maximum amplitude of the wavelike photometric variations in $V$ band (Strassmeier et al. 1993). The variations are shown for temperatures derived from $(B-V)$ (left-hand panels), $(V-R)$ (middle panels), and $(V-I)$ data (right-hand panels).

Reddy, B. E., Tomkin, J., Lambert, D. L., \& Allende Prieto, C. 2003, MNRAS, 340,304

Strassmeier, K. G., Hall, D. S., Fekel, F. C., \& Scheck, M. 1993, A\&AS, 100, 173 